\title{
Bond-coating in plasma-sprayed calcium-phosphate coatings
}

F. N. Oktar - M. Yetmez - S. Agathopoulos •

T. M. Lopez Goerne • G. Goller · I. Peker •

J. M. F. Ferreira

(C) Springer Science + Business Media, LLC 2007

\begin{abstract}
In the online and printed versions of the original article, there was a typographical error in I. Peker's name. His name is spelled correctly above. This error occurred in both the html and pdf versions of the online article.
\end{abstract}

The online version of the original article can be found at http://dx.doi.org/10.1007/s10856-006-0544-5. 